\title{
The Effective Dielectric Tensor for Electromagnetic Waves in Inhomogeneous Magnetized Plasmas and the Proper Formulation in the Electrostatic Limit
}

\author{
L. F. Ziebell and R. S. Schneider \\ Instituto de Física, UFRGS, Caixa Postal 15051, 91501-970 Porto Alegre, RS, Brazil
}

Received on 26 January, 2004

\begin{abstract}
The procedures used to obtain general expressions for the components of the effective dielectric tensor for electromagnetic waves in inhomogeneous magnetized plasmas are briefly reviewed, and the relationship between these expressions and their counterparts which can be obtained assuming electrostatic fluctuations is discussed. It is argued that a general formulation formerly available in the literature, which do not satisfy Onsager symmetry in the case of electrostatic fluctuations, is not the suitable form for description of dielectric properties in the electrostatic case, which require a dielectric constant. A general expression for an effective dielectric constant is therefore provided, obtained from the effective dielectric tensor, which satisfy Onsager symmetry.
\end{abstract}

\section{Introduction}

The subject of wave propagation in inhomogeneous plasmas can not be considered a simple problem, from a mathematical point of view. In a collisionless plasma it involves the use of Maxwell's equations for the components of the electromagnetic field, coupled to a set of Vlasov equations for the distributions of each of the plasma species. However, for the case of small amplitude perturbations with wavelengths much smaller than the scalelengths of inhomogeneities, the analysis can be simplified. In the simplest approach the fluctuations are described by a plane wave approximation, and a local relationship is assumed between current density and electric field. The procedure generates a dispersion relation which for a given wave frequency provides the local refraction index. Since the dispersion relation depends on local parameters, the approach is called the "locally homogeneous approximation".

Inhomogeneity effects can be explicitly included in the description of dielectric effects by simply taking into account in the evaluation of the dielectric tensor components the space derivatives of parameters that describe the plasma at each point, and then inserting these components into the same form of the dispersion relation utilized in the locally homogeneous approximation [1, 2, 3, 4]. However, this simple approach for the introduction of inhomogeneity effects has an important drawback. It leads to expressions for the components of the dielectric tensor which in general do not satisfy Onsager symmetry, except for wave propagation perpendicular to the direction of inhomogeneity [5]. As a consequence, non-resonant contributions appear to the antiHermitian parts of the components of the dielectric tensor, which means that the dielectric tensor obtained according to this approach do not properly describe the dielectric properties of the inhomogeneous medium and the exchange of energy between wave and particles.

The undesirable features of the dielectric tensor obtained with the plane wave approximation can be corrected by the introduction of the effective dielectric tensor, which is originated from an iterative procedure applied to the wave equation [6]. The procedure which leads to the effective dielectric tensor, starting from the conventional tensor derived with use of the plane wave approximation, will be called in what follows as BGI procedure, after the names of Beskin, Gurevich and Istomin, the authors of Ref. [6]. The BGI procedure assumes weak gradients in the physical parameters and neglects mode conversion and reflection. These simplifying assumptions may be violated near resonances or cut-offs, or for parameters for which two dispersion curves approach each other. However, many interesting phenomena occur in parameter regions with weak gradients where the WKB approximation is justified and where the effective dielectric tensor may play important role, as can be seen in a recent publication on geometrical optics [7].

We have early on been atracted by the potential usefulness of the concept of effective dielectric tensor and used it for several applications in magnetized plasmas, considering situations where the magnetic field is homogeneous and other parameters are inhomogeneous [5], situations where the magnetic field is inhomogeneous $[8,9]$, and situations where density and magnetic field inhomogeneities are present simultaneously $[10,11]$. In all these cases, we have obtained expressions which clearly satisfy Onsager symmetry and used them to obtain the solutions of the dispersion relation. It is important to remark the point about the Onsager symmetry, since although the general conception of the effective dielectric tensor is aimed to satisfy energy conservation, the proper symmetry of the tensor might be lost due to approximations introduced in the process of actual calculation of specific expressions of its components. If On- 
sager symmetry is satisfied, the anti-Hermitian parts of the dielectric tensor only feature resonant parts, properly describing wave-particle energy exchange. As it is known, if non-resonant terms appear in the anti-Hermitian parts of the dielectric tensor, they describe the variation of the wave amplitude due to the modification of the group velocity in an inhomogeneous medium, not true absorption or amplification $[12,13]$. This point has been illustrated with examples in Ref. [8], where results obtained from the dispersion relation with use of the effective dielectric tensor are compared with results obtained using other approaches found in the literature $[3,14,15,16,12]$.

The Onsager symmetry of the effective dielectric tensor for electromagnetic waves has also been considered in Ref. [17], for the case of homogeneous magnetic field. In fact, Ref. [17] obtained expressions which satisfy Onsager symmetry for the case of electromagnetic waves, corroborating our earlier results presented in Ref. [5]. However, Ref. [17] also considered the case of electrostatic waves, concluding that in this particular case the Onsager symmetry is not satisfied by the effective dielectric tensor. This is a particularly puzzling feature, which motivates the present investigation.

In the present paper we therefore return to the subject, with the objective of briefly review the derivation of the effective dielectric tensor for electromagnetic waves, and discuss its extension for the case of electrostatic waves. It is therefore sufficient to consider for simplicity the case of inhomogeneous density in homogeneous magnetic field. Inhomogeneities in other plasma parameters, like temperature, and in the magnetic field, could be also considered, but they are not essential to the point which we wish to demonstrate, and would only contribute to the complexity of the expressions for the components of the tensor. The calculations which follow show that the non-symmetrical tensor obtained when following the procedure indicated in Ref. [17] is not the correct form to be used for the study of electrostatic fluctuations. We therefore point out that the proper dispersion relation for electrostatic waves can be obtained from the effective dielectric tensor for electromagnetic waves, which features adequated symmetry properties. For the derivation, we consider a weakly inhomogeneous plasma with inhomogeneities along $x$ direction, immersed in a homogeneous magnetic field along $z$ direction, $\mathbf{B}_{\mathbf{0}}=B_{0} \mathbf{e}_{3}$. We also assume waves propagating in an arbitrary direction relative to ambient the magnetic field and to the inhomogeneity, with wave vector

$$
\mathbf{k}=k_{\perp} \cos \psi \mathbf{e}_{\mathbf{1}}+k_{\perp} \sin \psi \mathbf{e}_{\mathbf{2}}+k_{\|} \mathbf{e}_{\mathbf{3}}
$$

The procedure starts with the calculation of the components $\varepsilon_{i j}^{0}$, which are the components of the usual dielectric tensor for homogeneous medium, obtained with a plane wave approximation, but including terms corresponding to the gradients of the plasma distribution function [1]. The effective dielectric tensor is then obtained by application of the following rule,

$$
\overleftrightarrow{\varepsilon}=\overleftrightarrow{\varepsilon}^{0}+\frac{i}{2} \frac{\partial^{2} \overleftrightarrow{\varepsilon}^{0}}{\partial k_{x} \partial x}
$$

In section 2 we briefly review the steps for derivation of the components $\varepsilon_{i j}^{0}$ for the case of electromagnetic (EM) waves, which are necessary for the derivations of the effective dielectric tensor, according to the BGI procedure. In section 3 we obtain the corresponding components $\varepsilon_{i j}$ of the effective dielectric tensor, using Eq. (2), and also discuss the case of electrostatic (ES) fluctuations, according to the approach adopted in Ref. [17]. In section 4 we discuss the Onsager symmetry of the effective dielectric tensor, the validity of the approach adopted in section 3 for the case of ES fluctuations, and the proper application to the ES limit. In Sect. 5 we state the main results of the paper.

\section{Calculation of the $\stackrel{\leftrightarrow}{\varepsilon}$ tensor}

Let us consider a plasma with several species of particles, each denoted by the index $\alpha$. Each species is described by a distribution function $f_{\alpha}(\mathbf{r}, \mathbf{p}, t)$, where $\mathbf{r}$ is position, $\mathbf{p}$ is momentum, and $t$ is the time. The distribution function is normalized as follows

$$
\int d^{3} p f_{\alpha}(\mathbf{r}, \mathbf{p}, t)=n_{\alpha}(\mathbf{r}, t)
$$

where $n_{\alpha}(\mathbf{r}, t)$ is the number of particles of type $\alpha$ by unit of volume, at position $\mathbf{r}$ and time $t$.

In the absence of collisions the behavior of the system is governed by the Vlasov-Maxwell system of equations. Considering small amplitude fluctuations such as the system can be linearized, using the method of characteristics to solve the Vlasov equation, and assuming plane-wave approximation, the perturbed distribution function can be given by the well known result,

$$
f_{\alpha_{\mathbf{k}, \omega}}=-q_{\alpha} \mathbf{A}_{\alpha} \cdot \mathbf{E}_{\mathbf{k}, \omega}
$$

where

$$
\begin{gathered}
\mathbf{A}_{\alpha} \equiv \int_{-\infty}^{0} \boldsymbol{\Theta}_{\alpha} e^{i(\mathbf{k} \cdot \mathbf{R}-\omega \tau)} d \tau \\
\boldsymbol{\Theta}_{\alpha}=\left(1-\frac{\mathbf{p}^{\prime} \cdot \mathbf{k}}{m_{\alpha} \gamma_{\alpha}^{\prime} \omega}\right) \nabla_{\mathbf{p}^{\prime}} f_{\alpha 0}+\left(\frac{\mathbf{k} \cdot \nabla_{\mathbf{p}^{\prime}} f_{\alpha 0}}{m_{\alpha} \gamma_{\alpha}^{\prime} \omega}\right) \mathbf{p}^{\prime},
\end{gathered}
$$

and

$$
\mathbf{R}=\mathbf{r}^{\prime}-\mathbf{r}, \quad \tau=t^{\prime}-t
$$

and where we have used Faraday's law to relate magnetic and electric field. The quantity $f_{\alpha 0}$ is the equilibrium distribution function, which is a function of the constants of motion,

$$
f_{\alpha_{0}}(\mathbf{r}, \mathbf{p}, t)=F_{\alpha}\left(p_{\perp}^{2}, p_{\|}, \bar{X}_{\alpha}\right),
$$

where $p_{\|}$and $p_{\perp}$ are, respectively, the momentum parallel and perpendicular to the magnetic field $\mathbf{B}_{0}$, and where

$$
\bar{X}_{\alpha}=x+\frac{p_{y}}{m_{\alpha} \Omega_{\alpha}}
$$

with $\Omega_{\alpha}=q_{\alpha} B_{0} /\left(m_{\alpha} c\right)$ being the cyclotron angular frequency of particles of type $\alpha$. 
Therefore, it easily follows that the components of vector $\mathbf{A}_{\alpha}$ are

$$
\begin{aligned}
A_{\alpha x}= & {\left[\varphi_{0}\left(F_{\alpha}\right)+\frac{k_{\perp} \sin \psi}{m_{\alpha} \gamma_{\alpha} \omega} \frac{p_{\perp}}{m_{\alpha} \Omega_{\alpha}} \frac{\partial F_{\alpha}}{\partial \bar{X}_{\alpha}}\right] I_{x} } \\
A_{\alpha y}= & -\frac{k_{\perp} \cos \psi}{m_{\alpha} \gamma_{\alpha} \omega} \frac{p_{\perp}}{m_{\alpha} \Omega_{\alpha}} \frac{\partial F_{\alpha}}{\partial \bar{X}_{\alpha}} I_{x}+\varphi_{0}\left(F_{\alpha}\right) I_{y} \\
& +\left(1-\frac{k_{\|} p_{\|}}{m_{\alpha} \gamma_{\alpha} \omega}\right) \frac{1}{m_{\alpha} \Omega_{\alpha}} \frac{\partial F_{\alpha}}{\partial \bar{X}_{\alpha}} I_{z} \\
A_{\alpha z}= & \frac{k_{\perp} \cos \psi}{m_{\alpha} \gamma_{\alpha} \omega} \mathcal{L}\left(F_{\alpha}\right) I_{x}+\frac{k_{\perp}}{m_{\alpha} \gamma_{\alpha} \omega} \mathcal{\operatorname { s i n }} \psi \\
+ & {\left[\frac{\partial F_{\alpha}}{\partial p_{\|}}+\left(\frac{k_{\perp} \sin \psi}{m_{\alpha} \Omega_{\alpha}} \frac{\partial F_{\alpha}}{\partial \bar{X}_{\alpha}}\right) \frac{p_{\|}}{m_{\alpha} \gamma_{\alpha} \omega}\right] I_{z} }
\end{aligned}
$$

where

$$
\begin{aligned}
\varphi_{0} & =\left(1-\frac{k_{\|} p_{\|}}{m_{\alpha} \gamma_{\alpha} \omega}\right) \frac{\partial}{\partial p_{\perp}}+\frac{k_{\|} p_{\perp}}{m_{\alpha} \gamma_{\alpha} \omega} \frac{\partial}{\partial p_{\|}} \\
\mathcal{L} & =p_{\|} \frac{\partial}{\partial p_{\perp}}-p_{\perp} \frac{\partial}{\partial p_{\|}} \\
I_{x} & =\int_{-\infty}^{0} \frac{p_{x}^{\prime}}{p_{\perp}} e^{i(\mathbf{k} \cdot \mathbf{R}-\omega \tau)} d \tau \\
I_{y} & =\int_{-\infty}^{0} \frac{p_{y}^{\prime}}{p_{\perp}} e^{i(\mathbf{k} \cdot \mathbf{R}-\omega \tau)} d \tau \\
I_{z} & =\int_{-\infty}^{0} e^{i(\mathbf{k} \cdot \mathbf{R}-\omega \tau)} d \tau .
\end{aligned}
$$

Using the expressions for the unperturbed orbits in the proposed geometry, the $I_{x}, I_{y}$ and $I_{z}$ integrals can be written as follows

$$
I_{i}=i \sum_{n=-\infty}^{+\infty} e^{i\left[b_{\alpha} \sin (\varphi-\psi)-n(\varphi-\psi)\right]} \frac{1}{D_{\alpha n}}\left(\frac{p_{\perp}}{p_{\|}}\right)^{\delta_{i z}} \pi_{i}
$$

where

$$
D_{\alpha n} \equiv \omega-\frac{k_{\|} p_{\|}}{m_{\alpha} \gamma_{\alpha}}-\frac{n \Omega_{\alpha}}{\gamma_{\alpha}},
$$

and where the $\pi_{i}$ are the components of the following auxiliary vector,

$$
\begin{gathered}
\vec{\pi}_{n \alpha}=\left(\frac{n J_{n}\left(b_{\alpha}\right)}{b_{\alpha}} \cos \psi-i J_{n}^{\prime}\left(b_{\alpha}\right) \sin \psi\right) \mathbf{e}_{\mathbf{1}} \\
+\left(\frac{n J_{n}\left(b_{\alpha}\right)}{b_{\alpha}} \sin \psi+i J_{n}^{\prime}\left(b_{\alpha}\right) \cos \psi\right) \mathbf{e}_{\mathbf{2}} \\
+\frac{p_{\|}}{p_{\perp}} J_{n}\left(b_{\alpha}\right) \mathbf{e}_{\mathbf{3}}
\end{gathered}
$$

with $b_{\alpha}=k_{\perp} p_{\perp} /\left(m_{\alpha} \Omega_{\alpha}\right)$.

We now consider the distribution function. For a weakly inhomogeneous plasma it can be expanded as follows,

$$
F_{\alpha}\left(p_{\perp}^{2}, p_{\|}, \bar{X}_{\alpha}\right) \simeq f_{\alpha}\left(p_{\perp}^{2}, p_{\|}, x\right)+\left(\bar{X}_{\alpha}-x\right) f_{\alpha}^{\prime},
$$

where $f_{\alpha}\left(p_{\perp}^{2}, p_{\|}, x\right)$ is a local distribution with azimuthal symmetry, and $f_{\alpha}^{\prime}$ represents its derivative with respect the variable $x$.

Using Eq. (12), we obtain

$$
\begin{gathered}
A_{\alpha x}=\left[\varphi_{0}\left(f_{\alpha}\right)+\frac{p_{\perp} \sin \varphi}{m_{\alpha} \Omega_{\alpha}} \varphi_{0}\left(f_{\alpha}^{\prime}\right)+\frac{k_{\perp} \sin \psi}{m_{\alpha} \gamma_{\alpha} \omega} \frac{p_{\perp}}{m_{\alpha} \Omega_{\alpha}} f_{\alpha}^{\prime}\right] I_{x} \\
A_{\alpha y}=-\frac{k_{\perp} \cos \psi}{m_{\alpha} \gamma_{\alpha} \omega} \frac{p_{\perp}}{m_{\alpha} \Omega_{\alpha}} f_{\alpha}^{\prime} I_{x}+\left[\varphi_{0}\left(f_{\alpha}\right)+\frac{p_{\perp} \sin \varphi}{m_{\alpha} \Omega_{\alpha}} \varphi_{0}\left(f_{\alpha}^{\prime}\right)\right] I_{y} \\
+\left(1-\frac{k_{\|} p_{\|}}{m_{\alpha} \gamma_{\alpha} \omega}\right) \frac{1}{m_{\alpha} \Omega_{\alpha}} f_{\alpha}^{\prime} I_{z} \\
A_{\alpha z}=\frac{k_{\perp} \cos \psi}{m_{\alpha} \gamma_{\alpha} \omega}\left[\mathcal{L}\left(f_{\alpha}\right)+\frac{p_{\perp} \sin \varphi}{m_{\alpha} \Omega_{\alpha}} \mathcal{L}\left(f_{\alpha}^{\prime}\right)\right] I_{x} \\
+\frac{k_{\perp} \sin \psi}{m_{\alpha} \gamma_{\alpha} \omega}\left[\mathcal{L}\left(f_{\alpha}\right)+\frac{p_{\perp} \sin \varphi}{m_{\alpha} \Omega_{\alpha}} \mathcal{L}\left(f_{\alpha}^{\prime}\right)\right] I_{y} \\
+\left[\frac{\partial f_{\alpha}}{\partial p_{\|}}+\frac{p_{\perp} \sin \varphi}{m_{\alpha} \Omega_{\alpha}} \frac{\partial f_{\alpha}^{\prime}}{\partial p_{\|}}+\frac{k_{\perp} \sin \psi}{m_{\alpha} \gamma_{\alpha} \omega} \frac{p_{\|}}{m_{\alpha} \Omega_{\alpha}} f_{\alpha}^{\prime}\right] I_{z} .
\end{gathered}
$$

These expressions are used to obtain the perturbed distribution function, according to Eq. (4). The distribution is then utilized to evaluate the current, generating a linear relationship, possibly anisotropic, between the Fourier trans- forms of current and electric field,

$$
\mathbf{J}_{\mathbf{k}, \omega}=\stackrel{\leftrightarrow}{\sigma}^{0}(\mathbf{k}, \omega ; x) \cdot \mathbf{E}_{\mathbf{k}, \omega}
$$


It is important to remark that the tensor $\stackrel{\leftrightarrow}{\sigma}^{0}$ is not the Fourier transform of the conductivity tensor in configuration space, which relates current density and electric field in a stationary and inhomogeneous medium

$$
\mathbf{J}(\mathbf{r}, t)=\int d^{3} x^{\prime} \int d t \stackrel{\leftrightarrow}{\sigma}\left(\mathbf{r}, \mathbf{r}^{\prime}, t-t^{\prime}\right) \cdot \mathbf{E}\left(\mathbf{r}^{\prime}, t^{\prime}\right),
$$

since a relationship like Eq. (16) only would be obtained by Fourier transform from Eq. (17) in the case of a homogeneous medium, granting the use of the convolution theorem.

Using the expression obtained for the $\sigma_{i j}^{0}$, and following the usual procedures, one arrives to the following expression for the components of the plane wave dielectric tensor:

$$
\varepsilon_{i j}^{0}=\delta_{i x}-i \omega \sum_{\alpha} \frac{X_{\alpha}}{n_{\alpha}} \int d^{3} p \frac{1}{\gamma_{\alpha}} p_{i} A_{\alpha_{j}},
$$

where

$$
X_{\alpha}=\frac{\omega_{p \alpha}^{2}}{\omega^{2}}, \quad \text { and where } \omega_{p \alpha}=\left(\frac{4 \pi n_{\alpha} q_{\alpha}^{2}}{m_{\alpha}}\right)^{1 / 2}
$$

is the plasma frequency for the particles of species $\alpha$. Using in Eq. (18) the expressions given by Eqs. (13) to (15), along with Eq. (9), and following through some tedious algebra, we obtain

$$
\varepsilon_{i j}^{0}=\varepsilon_{i j}^{0 h}+\varepsilon_{i j}^{0 n h}
$$

where

$$
\begin{gathered}
\varepsilon_{i j}^{0 h}=\delta_{i j}+\omega \sum_{\alpha} \frac{X_{\alpha}}{n_{\alpha}} \sum_{n=-\infty}^{+\infty} \int d^{3} p p_{\perp} \frac{1}{\gamma_{\alpha} D_{\alpha n}} \pi_{i}^{*} \\
\times\left\{\left(1-\delta_{j z}\right) \varphi_{0}\left(f_{\alpha}\right) \pi_{j}+\delta_{j z}\left[\frac{k_{\perp}}{m_{\alpha} \gamma_{\alpha} \omega} \frac{n}{b_{\alpha}} \mathcal{L}\left(f_{\alpha}\right)+\frac{\partial f_{\alpha}}{\partial p_{\|}}\right] \frac{p_{\perp}}{p_{\|}} \pi_{z}\right\}, \\
\varepsilon_{i j}^{0 n h}=\omega \sum_{\alpha} \frac{X_{\alpha}}{n_{\alpha}} \frac{1}{m_{\alpha} \Omega_{\alpha}} \sum_{n=-\infty}^{+\infty} \int d^{3} p p_{\perp}^{2} \frac{1}{\gamma_{\alpha} D_{\alpha n}} \\
\times\left\{\delta_{j y}\left[\left(1-\frac{k_{\|} p_{\|}}{m_{\alpha} \gamma_{\alpha} \omega}\right)-\frac{k_{\perp} p_{\perp}}{m_{\alpha} \gamma_{\alpha} \omega} \frac{n}{b_{\alpha}}\right] \frac{1}{p_{\|}} f_{\alpha}^{\prime} \pi_{i}^{*} \pi_{z}\right. \\
+\frac{k_{\perp} \sin \psi}{m_{\alpha} \gamma_{\alpha} \omega} f_{\alpha}^{\prime} \pi_{i}^{*} \pi_{j}+\left(1-\delta_{j z}\right) \varphi_{0}\left(f_{\alpha}^{\prime}\right) \Phi_{i}^{*} \pi_{j} \\
\left.+\delta_{j z}\left[\frac{k_{\perp}}{m_{\alpha} \gamma_{\alpha} \omega} \frac{n}{b_{\alpha}} \mathcal{L}\left(f_{\alpha}^{\prime}\right)+\frac{\partial f_{\alpha}^{\prime}}{\partial p_{\|}}\right] \frac{p_{\perp}}{p_{\|}} \Phi_{i}^{*} \pi_{z}\right\} .
\end{gathered}
$$

In the derivation of these expressions, we have used

$$
\begin{aligned}
J_{i} & =\int_{0}^{2 \pi} p_{i} e^{i\left[b_{\alpha} \sin (\varphi-\psi)-n(\varphi-\psi)\right]} d \varphi=2 \pi p_{\perp} \pi_{i}^{*} \\
K_{i} & =\int_{0}^{2 \pi} p_{i} \sin \varphi e^{i\left[b_{\alpha} \sin (\varphi-\psi)-n(\varphi-\psi)\right]} d \varphi=2 \pi p_{\perp} \Phi_{i}^{*}
\end{aligned}
$$

where the $\Phi_{i}$ are components of the following vector

$$
\begin{gathered}
\vec{\Phi}_{n \alpha}=\left\{\left[\left(\frac{n^{2}}{b_{\alpha}^{2}}-\frac{1}{2}\right) J_{n}\left(b_{\alpha}\right)-\frac{J_{n}^{\prime}\left(b_{\alpha}\right)}{b_{\alpha}}\right] \sin (2 \psi)\right. \\
\left.-i\left[\frac{n}{b_{\alpha}^{2}} J_{n}\left(b_{\alpha}\right)-\frac{n J_{n}^{\prime}\left(b_{\alpha}\right)}{b_{\alpha}}\right] \cos (2 \psi)\right\} \mathbf{e}_{\mathbf{1}} \\
+\left\{\frac{J_{n}}{2}-\left[\left(\frac{n^{2}}{b_{\alpha}^{2}}-\frac{1}{2}\right) J_{n}\left(b_{\alpha}\right)-\frac{J_{n}^{\prime}\left(b_{\alpha}\right)}{b_{\alpha}}\right] \cos (2 \psi)\right. \\
\left.-i\left[\frac{n}{b_{\alpha}^{2}} J_{n}\left(b_{\alpha}\right)-\frac{n J_{n}^{\prime}\left(b_{\alpha}\right)}{b_{\alpha}}\right] \sin (2 \psi)\right\} \mathbf{e}_{\mathbf{2}} \\
+\frac{p_{\|}}{p_{\perp}}\left[\frac{n}{b_{\alpha}} J_{n}\left(b_{\alpha}\right) \sin \psi+i J_{n}^{\prime}\left(b_{\alpha}\right) \cos \psi\right] \mathbf{e}_{\mathbf{3}} .
\end{gathered}
$$


An alternative form for these expressions can be obtained if we take into account the definition of $b_{\alpha}$ and cancel out the $k_{\perp}$ which appear in the combination $k_{\perp} / b_{\alpha}$, in Eqs. (20) and (21). We arrive to the following form,

$$
\begin{aligned}
\varepsilon_{i j}^{0 h}=\delta_{i j}+ & \omega \sum_{\alpha} \frac{X_{\alpha}}{n_{\alpha}} \sum_{n=-\infty}^{+\infty} \int d^{3} p p_{\perp} \frac{1}{\gamma_{\alpha} D_{\alpha n}} \varphi_{0}\left(f_{\alpha}\right) \pi_{i}^{*} \pi_{j} \\
& -\delta_{i z} \delta_{j z} \sum_{\alpha} \frac{X_{\alpha}}{n_{\alpha}} \int d^{3} p \frac{1}{\gamma_{\alpha}} \frac{p_{\|}}{p_{\perp}} \mathcal{L}\left(f_{\alpha}\right) \\
\varepsilon_{i j}^{0 n h}= & \omega \sum_{\alpha} \frac{X_{\alpha}}{n_{\alpha}} \frac{1}{m_{\alpha} \Omega_{\alpha}} \sum_{n=-\infty}^{+\infty} \int d^{3} p p_{\perp}^{2} \frac{1}{\gamma_{\alpha} D_{\alpha n}} \\
& \times\left[\frac{k_{\perp} \sin \psi}{m_{\alpha} \gamma_{\alpha} \omega} f_{\alpha}^{\prime} \pi_{i}^{*} \pi_{j}+\varphi_{0}\left(f_{\alpha}^{\prime}\right) \Phi_{i}^{*} \pi_{j}\right] \\
+\left(\delta_{i y} \delta_{j z}\right. & \left.+\delta_{j y} \delta_{i z}\right) \sum_{\alpha} \frac{X_{\alpha}}{n_{\alpha}} \frac{1}{m_{\alpha} \Omega_{\alpha}} \int d^{3} p p_{\perp} \frac{1}{\gamma_{\alpha}} \frac{p_{\|}}{p_{\perp}} f_{\alpha}^{\prime} .
\end{aligned}
$$

Details about this transformation can be found in Appendix A. The $\varepsilon_{i j}^{0}$ components given by Eqs. (25) and (26) are exactly equivalent to those appearing in Ref. [5], since it can easily be demonstrated that

$$
\begin{gathered}
\pi_{i}^{*} \pi_{j}=\left(\frac{p_{\|}}{p_{\perp}}\right)^{\delta_{i z}+\delta_{j z}} R_{i j} \\
\Phi_{i}^{*} \pi_{j}=\left(\frac{p_{\|}}{p_{\perp}}\right)^{\delta_{i z}+\delta_{j z}} S_{i j},
\end{gathered}
$$

where the $R_{i j}$ and the $S_{i j}$ are those defined in Ref. [5].

Eq. (25), as well as Eq. (20), are the conventional expressions for the dielectric tensor obtained with the plane wave approximation. Eqs. (26) and (21) are alternative formulations for the contribution originated when weak inhomogeneities are taken into account, which have to be added to the homogeneous contribution. As mentioned in the Introduction section, the resulting dielectric tensor do not satisfy Onsager symmetry.

\section{Calculation of the effective dielec- tric tensor, $\overleftrightarrow{\varepsilon}$}

The tensor $\overleftrightarrow{\varepsilon}$ for the general case of EM fluctuations may be now obtained by application of Eq. (2) to Eq. (18). Using the formulation given by Eqs. (20) and (21), we obtain the following correction for each $i j$ component of the tensor,

$$
\begin{gathered}
\frac{i}{2} \frac{\partial^{2}}{\partial k_{x} \partial x} \varepsilon_{i j}^{0 h}=\frac{i}{2}\left[\omega \sum_{\alpha} \frac{X_{\alpha}}{n_{\alpha}} \sum_{n=-\infty}^{+\infty} \int d^{3} p p_{\perp} \frac{1}{\gamma_{\alpha} D_{\alpha_{n}}}\right. \\
\times\left\{\left(1-\delta_{j z}\right) \varphi_{0}\left(f_{\alpha}^{\prime}\right) \frac{\partial}{\partial k_{x}}\left(\pi_{i}^{*} \pi_{j}\right)\right. \\
\left.\left.+\delta_{j z}\left[\frac{k_{\perp}}{m_{\alpha} \gamma_{\alpha} \omega} \frac{n}{b_{\alpha}} \mathcal{L}\left(f_{\alpha}^{\prime}\right)+\frac{\partial f_{\alpha}^{\prime}}{\partial p_{\|}}\right] \frac{p_{\perp}}{p_{\|}} \frac{\partial}{\partial k_{x}}\left(\pi_{i}^{*} \pi_{z}\right)\right\}\right],
\end{gathered}
$$

where we notice that the quantity

$$
\frac{k_{\perp}}{m_{\alpha} \gamma_{\alpha} \omega} \frac{n}{b_{\alpha}}
$$

is independent of $k_{x}$ because of the $k_{\perp}$ dependence of $b_{\alpha}$, and therefore don't need to be derived.

The derivative of the product $\pi_{i}^{*} \pi_{j}$ can be written in terms of the components $\pi_{i}$ themselves and components $\Phi_{i}$,

$$
\frac{\partial}{\partial k_{x}}\left(\pi_{i}^{*} \pi_{j}\right)=\frac{p_{\perp}}{m_{\alpha} \Omega_{\alpha}} i\left(\Phi_{i}^{*} \pi_{j}-\Phi_{j} \pi_{i}^{*}\right) .
$$

Using this equation and adding the corrections obtained to the $\varepsilon_{i j}^{0}$ components, we readily arrive to the expression for the components of the effective dielectric tensor

$$
\begin{gathered}
\varepsilon_{i j}=\varepsilon_{i j}^{0 h}+\varepsilon_{i j}^{0 n h}+\frac{i}{2} \frac{\partial^{2}}{\partial k_{x} \partial x} \varepsilon_{i j}^{0 h} \\
=\delta_{i j}+\omega \sum_{\alpha} \frac{X_{\alpha}}{n_{\alpha}} \sum_{n=-\infty}^{+\infty} \int d^{3} p p_{\perp} \frac{1}{\gamma_{\alpha} D_{\alpha_{n}}} \pi_{i}^{*} \\
\times\left\{\left(1-\delta_{j z}\right) \varphi_{0}\left(f_{\alpha}\right) \pi_{j}+\delta_{j z}\left[\frac{k_{\perp}}{m_{\alpha} \gamma_{\alpha} \omega} \frac{n}{b_{\alpha}} \mathcal{L}\left(f_{\alpha}\right)+\frac{\partial f_{\alpha}}{\partial p_{\|}}\right] \frac{p_{\perp}}{p_{\|}} \pi_{z}\right\}
\end{gathered}
$$




$$
\begin{gathered}
+\omega \sum_{\alpha} \frac{X_{\alpha}}{n_{\alpha}} \frac{1}{m_{\alpha} \Omega_{\alpha}} \sum_{n=-\infty}^{+\infty} \int d^{3} p p_{\perp}^{2} \frac{1}{\gamma_{\alpha} D_{\alpha_{n}}} \\
\times\left\{\delta_{j y}\left[\left(1-\frac{k_{\|} p_{\|}}{m_{\alpha} \gamma_{\alpha} \omega}\right)-\frac{k_{\perp} p_{\perp}}{m_{\alpha} \gamma_{\alpha} \omega} \frac{n}{b_{\alpha}}\right] \frac{1}{p_{\|}} f_{\alpha}^{\prime} \pi_{i}^{*} \pi_{z}\right. \\
+\frac{k_{\perp} \sin \psi}{m_{\alpha} \gamma_{\alpha} \omega} f_{\alpha}^{\prime} \pi_{i}^{*} \pi_{j}+\left(1-\delta_{j z}\right) \varphi_{0}\left(f_{\alpha}^{\prime}\right)\left(\Phi_{i}^{*} \pi_{j}\right)^{H} \\
\left.+\delta_{j z}\left[\frac{k_{\perp}}{m_{\alpha} \gamma_{\alpha} \omega} \frac{n}{b_{\alpha}} \mathcal{L}\left(f_{\alpha}^{\prime}\right)+\frac{\partial f_{\alpha}^{\prime}}{\partial p_{\|}}\right] \frac{p_{\perp}}{p_{\|}}\left(\Phi_{i}^{*} \pi_{z}\right)^{H}\right\} .
\end{gathered}
$$

As in the case of the plane wave tensor, an alternative form of the effective tensor can be obtained by cancelling out the $k_{\perp}$ which appear in numerator and denominator in the combination $k_{\perp} / b_{\alpha}$ (details in Appendix A). After this operation, the components of the effective dielectric tensor are written as follows

$$
\begin{aligned}
\varepsilon_{i j}=\delta_{i j} & +\omega \sum_{\alpha} \frac{X_{\alpha}}{n_{\alpha}} \sum_{n=-\infty}^{+\infty} \int d^{3} p p_{\perp} \frac{1}{\gamma_{\alpha} D_{\alpha_{n}}} \varphi_{0}\left(f_{\alpha}\right) \pi_{i}^{*} \pi_{j} \\
& -\delta_{i z} \delta_{j z} \sum_{\alpha} \frac{X_{\alpha}}{n_{\alpha}} \int d^{3} p \frac{1}{\gamma_{\alpha}} \frac{p_{\|}}{p_{\perp}} \mathcal{L}\left(f_{\alpha}\right) \\
+ & \delta_{j y} \delta_{i z} \sum_{\alpha} \frac{X_{\alpha}}{n_{\alpha}} \frac{1}{m_{\alpha} \Omega_{\alpha}} \int d^{3} p p_{\perp} \frac{1}{\gamma_{\alpha}} \frac{p_{\|}}{p_{\perp}} f_{\alpha}^{\prime} \\
+ & \omega \sum_{\alpha} \frac{X_{\alpha}}{n_{\alpha}} \frac{1}{m_{\alpha} \Omega_{\alpha}} \sum_{n=-\infty}^{+\infty} \int d^{3} p p_{\perp}^{2} \frac{1}{\gamma_{\alpha} D_{\alpha_{n}}} \\
& \times\left[\frac{k_{\perp} \sin \psi}{m_{\alpha} \gamma_{\alpha} \omega} f_{\alpha}^{\prime} \pi_{i}^{*} \pi_{j}+\varphi_{0}\left(f_{\alpha}^{\prime}\right)\left(\Phi_{i}^{*} \pi_{j}\right)^{H}\right] \\
-\delta_{j z} \sum_{\alpha} & \frac{X_{\alpha}}{n_{\alpha}} \frac{1}{m_{\alpha} \Omega_{\alpha}} \sum_{n=-\infty}^{+\infty} \int d^{3} p p_{\perp} \frac{1}{\gamma_{\alpha}} \frac{p_{\perp}}{p_{\|}} \mathcal{L}\left(f_{\alpha}^{\prime}\right)\left(\Phi_{i}^{*} \pi_{j}\right)^{H} .
\end{aligned}
$$

Now we take into account the following property, which can be easily demonstrated,

$$
\sum_{n=-\infty}^{+\infty}\left(\Phi_{i}^{*} \pi_{z}\right)^{H}=\frac{1}{2} \frac{p_{\|}}{p_{\perp}} \delta_{i y},
$$

and write the last term in Eq. (31) as follows

$$
\begin{gathered}
-\delta_{i y} \delta_{j z} \frac{1}{2} \sum_{\alpha} \frac{X_{\alpha}}{n_{\alpha}} \frac{1}{m_{\alpha} \Omega_{\alpha}} \int d^{3} p p_{\perp} \frac{1}{\gamma_{\alpha}} \mathcal{L}\left(f_{\alpha}^{\prime}\right) \\
=\delta_{i y} \delta_{j z} \sum_{\alpha} \frac{X_{\alpha}}{n_{\alpha}} \frac{1}{m_{\alpha} \Omega_{\alpha}} \int d^{3} p \frac{1}{\gamma_{\alpha}} p_{\|} f_{\alpha}^{\prime},
\end{gathered}
$$

where the last step was obtained via integration by parts.

Using this result in Eq. (31),

$$
\begin{aligned}
\varepsilon_{i j}=\delta_{i j} & +\omega \sum_{\alpha} \frac{X_{\alpha}}{n_{\alpha}} \sum_{n=-\infty}^{+\infty} \int d^{3} p p_{\perp} \frac{1}{\gamma_{\alpha} D_{\alpha_{n}}} \varphi_{0}\left(f_{\alpha}\right) \pi_{i}^{*} \pi_{j} \\
& -\delta_{i z} \delta_{j z} \sum_{\alpha} \frac{X_{\alpha}}{n_{\alpha}} \int d^{3} p \frac{1}{\gamma_{\alpha}} \frac{p_{\|}}{p_{\perp}} \mathcal{L}\left(f_{\alpha}\right) \\
+ & \omega \sum_{\alpha} \frac{X_{\alpha}}{n_{\alpha}} \frac{1}{m_{\alpha} \Omega_{\alpha}} \sum_{n=-\infty}^{+\infty} \int d^{3} p p_{\perp}^{2} \frac{1}{\gamma_{\alpha} D_{\alpha_{n}}} \\
\times & {\left[\frac{k_{\perp} \sin \psi}{m_{\alpha} \gamma_{\alpha} \omega} f_{\alpha}^{\prime} \pi_{i}^{*} \pi_{j}+\varphi_{0}\left(f_{\alpha}^{\prime}\right)\left(\Phi_{i}^{*} \pi_{j}\right)^{H}\right] } \\
+\left(\delta_{i y} \delta_{j z}\right. & \left.+\delta_{j y} \delta_{i z}\right) \sum_{\alpha} \frac{X_{\alpha}}{n_{\alpha}} \frac{1}{m_{\alpha} \Omega_{\alpha}} \int d^{3} p p_{\perp} \frac{1}{\gamma_{\alpha}} \frac{p_{\|}}{p_{\perp}} f_{\alpha}^{\prime}
\end{aligned}
$$


These expressions for the components of the effective dielectric tensor are exactly equivalent to those obtained in Ref. [5].

According to the approach adopted in Ref. [17], we proceed to derive expressions for the dielectric tensor for the case of ES fluctuations. The components of the effective dielectric tensor in the electrostatic limit can be obtained from those which we have derived for EM waves by assumption of vanishing value of the components of the wave vector appearing in the numerators of the differential operator applied to the distribution function. These components originated from the use of Faraday's law for the magnetic fluctuations, $\mathbf{k} \times \mathbf{E}=(\omega / c) \mathbf{B}$. It is clear that the more familiar form given by Eq. (33) of course can not be used for obtaining this limit, because some of these $k_{i}$ components have been cancelled out and are no longer present. However, Eq. (30) can be used for such a procedure, and after taking the limit of vanishing $k_{i}$ in the differential operator we obtain the following limiting expression for the components of the effective dielectric tensor,

$$
\begin{gathered}
\varepsilon_{i j}=\delta_{i j}+\omega \sum_{\alpha} \frac{X_{\alpha}}{n_{\alpha}} \sum_{n=-\infty}^{+\infty} \int d^{3} p p_{\perp} \frac{1}{\gamma_{\alpha} D_{\alpha_{n}}} \\
\times\left\{\frac{\partial f_{\alpha}}{\partial p_{\perp}} \pi_{i}^{*} \pi_{j}-\delta_{j z} \frac{1}{p_{\|}} \mathcal{L}\left(f_{\alpha}\right) \pi_{i}^{*} \pi_{z}\right\} \\
+\omega \sum_{\alpha} \frac{X_{\alpha}}{n_{\alpha}} \frac{1}{m_{\alpha} \Omega_{\alpha}} \sum_{n=-\infty}^{+\infty} \int d^{3} p p_{\perp}^{2} \frac{1}{\gamma_{\alpha} D_{\alpha_{n}}} \\
\times\left[\delta_{j y} \frac{1}{p_{\|}} f_{\alpha}^{\prime} \pi_{i}^{*} \pi_{z}+\frac{\partial f_{\alpha}^{\prime}}{\partial p_{\perp}}\left(\Phi_{i}^{*} \pi_{j}\right)^{H}-\delta_{j z} \frac{1}{p_{\|}} \mathcal{L}\left(f_{\alpha}^{\prime}\right)\left(\Phi_{i}^{*} \pi_{z}\right)^{H}\right] .
\end{gathered}
$$

Of course, the procedure of assuming vanishing values of the components $k_{i}$ in the differential operator applied to the distribution function, in order to obtain the ES limit, is only an useful artificial procedure. It is equivalent to assuming as a starting condition that magnetic fluctuations do not exist. In that case, the quantity $\Theta_{\alpha}$ which was defined along with Eq. (4) would be simply $\Theta_{\alpha}=\nabla_{\mathbf{p}^{\prime}} f_{\alpha 0}$, and Eqs. (13), (14), and (15) would be reduced simply to

$$
A_{\alpha x}=\frac{\partial f_{\alpha}}{\partial p_{\perp}}, \quad A_{\alpha y}=\frac{\partial f_{\alpha}}{\partial p_{\perp}}, \quad A_{\alpha z}=\frac{\partial f_{\alpha}}{\partial p_{\|}} .
$$

All other procedures would be exactly as described in the more general case of EM waves, and the outcome would be exactly Eq. (34).

\section{The symmetry properties of the ef- fective dielectric tensor and the ap- plication to the electrostatic limit}

The effective dielectric tensor as given by Eq. (33) features proper Onsager symmetry [5], as it is easily verified by considering that it contains the Hermitean part of $\Phi_{i}^{*} \pi_{j}$, and that the product $\pi_{i}^{*} \pi_{j}$ is also Hermitean. When written in the form of Eq. (30), however, the symmetry of the effective dielectric tensor is apparently not satisfied, due to the term with $\delta_{j y}$ and $\delta_{j z}$. However, despite the apparent asymmetry, the tensor in the form of Eq. (30) also satisfies Onsager symmetry, since it is equivalent to Eq. (33).

When considering the ES limit, however, an important feature emerges. The terms originated from the magnetic field fluctuations, containing the $k_{i}$ components in the numerator of the differential operator, were essential in the transformation between Eq. (30) and Eq. (33), as can be seen in Appendix A. In the case of the ES limit, when these terms are no longer present, the same operation which makes the symmetry evident can not be made. As a result, by considering the components of the tensor in the ES limit, Eq. (34), we obtain the following result

$$
\begin{gathered}
\varepsilon_{i j}-\varepsilon_{j i}^{*}=-\omega \sum_{\alpha} \frac{X_{\alpha}}{n_{\alpha}} \sum_{n=-\infty}^{+\infty} \int d^{3} p p_{\perp} \frac{1}{\gamma_{\alpha} D_{\alpha_{n}}} \\
\times \frac{1}{p_{\|}} \mathcal{L}\left(f_{\alpha}\right)\left[\delta_{j z} \pi_{i}^{*} \pi_{z}-\delta_{i z} \pi_{j} \pi_{z}\right] \\
+\omega \sum_{\alpha} \frac{X_{\alpha}}{n_{\alpha}} \frac{1}{m_{\alpha} \Omega_{\alpha}} \sum_{n=-\infty}^{+\infty} \int d^{3} p p_{\perp}^{2} \frac{1}{\gamma_{\alpha} D_{\alpha_{n}}} \\
\times\left\{\frac{1}{p_{\|}} f_{\alpha}^{\prime}\left[\delta_{j y} \pi_{i}^{*} \pi_{z}-\delta_{i y} \pi_{j} \pi_{z}\right]\right. \\
\left.-\frac{1}{p_{\|}} \mathcal{L}\left(f_{\alpha}^{\prime}\right)\left[\delta_{j z}\left(\Phi_{i}^{*} \pi_{z}\right)^{H}-\delta_{i z}\left(\Phi_{j} \pi_{z}\right)^{H}\right]\right\} .
\end{gathered}
$$

It is seen that the quantities $\varepsilon_{i j}$ and $\varepsilon_{j i}^{*}$ have a nonvanishing difference in the ES limit, leading to the conclusion that the effective dielectric tensor do not satisfy Onsager symmetry when written in the ES limit, while the effective tensor derived for the general case of EM waves satisfies Onsager symmetry. This conclusion is the same as obtained in Ref. [17], where the lack of Onsager symmetry in 
the ES case was atributed to the diamagnetic current which occurs in the direction respectively perpendicular to the ambient magnetic field and to the inhomogeneity. However, in what follows we argue with more fundamental reasons to show that the so called 'dielectric tensor for ES waves' obtained in Ref. [17], and which has been discussed in the present paper as a limiting form, is not the correct way to deal with ES fluctuations, making meaningless and without consequence the lack of Onsager symmetry of the effective dielectric tensor in the ES limit.

First of all, we start by considering that the dielectric tensor given by Eqs. (30) and (33) features the proper symmetry and can be used in the dispersion relation for quite general circunstances, even for low level of magnetic perturbation. Let us therefore examine the dispersion relation. In the electrostatic approximation, $\mathbf{B}_{\mathbf{1}} \simeq 0$, and Gauss's law can be used to obtain the dispersion relation. Using it along with the equation of continuity, and using plane wave approximation for the electric field, we obtain the following

$$
i \mathbf{k} \cdot \mathbf{E}=-\frac{4 \pi i}{\omega}[(\nabla \cdot \overleftrightarrow{\sigma}) \cdot \mathbf{E}+i \mathbf{k} \cdot \overleftrightarrow{\sigma} \cdot \mathbf{E}]
$$

where we have used the relationship between the effective dielectric tensor and the effective conductivity,

$$
\varepsilon_{i j}=\delta_{i j}+\frac{4 \pi i}{\omega} \sigma_{i j}
$$

Considering now that in the ES limit $\mathbf{E} \simeq-\nabla \phi=$ $-i \mathbf{k} \phi$, we obtain the following form of the dispersion relation [18],

$$
k^{2} \varepsilon_{l}-i \mathbf{k} \cdot(\nabla \cdot \stackrel{\leftrightarrow}{\varepsilon})=0,
$$

where we have introduced the effective dielectric constant,

$$
\varepsilon_{l}=\sum_{i j} \frac{k_{i} \varepsilon_{i j} k_{j}}{k^{2}} .
$$

It is important to point out that the dielectric tensor to be used in Eq. (37) is the effective dielectric tensor, which is free from the inconsistencies arising from the use of the local approximation for inhomogeneous plasmas. We also point out that the term with derivatives of the components of the dielectric tensor introduces inhomogeneity effects which are of the same order of inhomogeneity effects which were taken into account in the derivation of the effective dielectric tensor. Even if it can be negligible in some circunstances [18], it has been shown to play essential role in the description of the lower hybrid drift instability (LHDI), an instability with strong electrostatic character which occurs in the lower hybrid range of frequencies [11].

For the actual derivation of the dielectric constant we start from the components of the effective dielectric tensor as given by Eq. (30), and obtain the following,

$$
\begin{gathered}
\varepsilon_{l}=1+\frac{\omega^{2}}{k^{2}} \sum_{\alpha} \frac{X_{\alpha}}{n_{\alpha}} m_{\alpha} \sum_{n=-\infty}^{+\infty} \int d^{3} p \frac{1}{D_{\alpha_{n}}} J_{n}^{2} \\
\times\left[k_{\perp} \frac{n}{b_{\alpha}} \frac{\partial f_{\alpha}}{\partial p_{\perp}}+k_{\|} \frac{\partial f_{\alpha}}{\partial p_{\|}}\right] \\
+\frac{\omega^{2}}{k^{2}} \sum_{\alpha} \frac{X_{\alpha}}{n_{\alpha}} \frac{k_{\perp}}{\Omega_{\alpha}} \sum_{n=-\infty}^{\sin \psi} \int d^{3} p \frac{1}{D_{\alpha_{n}}} J_{n}^{2} f_{\alpha}^{\prime} \\
+\frac{\omega^{2}}{k^{2}} \sum_{\alpha} \frac{X_{\alpha}}{n_{\alpha}} \frac{\sin \psi}{\Omega_{\alpha}} \sum_{n=-\infty}^{+\infty} \int d^{3} p p_{\perp} \frac{1}{D_{\alpha_{n}}} \\
\times\left[\frac{n J_{n}^{2}}{b_{\alpha}}-\frac{\Omega_{\alpha}}{\gamma_{\alpha} \omega} J_{n} J_{n}^{\prime}\right]\left[k_{\perp} \frac{n}{b_{\alpha}} \frac{\partial f_{\alpha}^{\prime}}{\partial p_{\perp}}+k_{\|} \frac{\partial f_{\alpha}^{\prime}}{\partial p_{\|}}\right] .
\end{gathered}
$$

Details about this calculation can be found in Appendix B. It is important to notice that all the $k_{i}$ components originated from the magnetic fluctuations have been cancelled out in the derivation of Eq. (39), so that the dielectric constant is the same as it would be obtained if we had started assuming electrostatic fluctuations when deriving the components of the dielectric tensor. This result was attained as a consequence of the double scalar product contained in $\mathbf{k} \cdot \overleftrightarrow{\varepsilon} \cdot \mathbf{k}$, without the need of considering any nonsymmetrical dielectric tensor,

By taking into account that in the present application the inhomogeneities are along the $x$ direction, evaluating the derivatives of the components of the dielectric tensor as given by Eq. (30), and using Eq. (39), the dispersion relation (37) can be written more explicitly as follows,

$$
\begin{gathered}
-i \frac{\omega}{k^{2}} \sum_{\alpha} \frac{X_{\alpha}}{n_{\alpha}} \sum_{n=-\infty}^{+\infty} \int d^{3} p p_{\perp} \frac{1}{\gamma_{\alpha} D_{\alpha_{n}}}\left[k_{\perp} \frac{n}{b_{\alpha}} \frac{\partial f_{\alpha}^{\prime}}{\partial p_{\perp}}+k_{\|} \frac{\partial f_{\alpha}^{\prime}}{\partial p_{\|}}\right] \\
\times\left(\frac{n J_{n}^{2}}{b_{\alpha}} \cos \psi+i J_{n} J_{n}^{\prime} \sin \psi\right)+\varepsilon_{l}=0,
\end{gathered}
$$

where the dielectric constant $\varepsilon_{l}$ is given by Eq. (39).

Some limiting cases can be now considered. For instance, in the case of waves propagating parallel to the ambient magnetic field $\left(k_{\perp}=0\right)$, we obtain the well known dispersion relation for electrostatic waves propagating along the ambient magnetic field,

$$
\varepsilon_{l}=1+\sum_{\alpha} \frac{4 \pi q_{\alpha}^{2}}{k_{\|}} \int d^{3} p \frac{1}{D_{\alpha_{0}}} \frac{\partial f_{\alpha}}{\partial p_{\|}}=0 .
$$

This expression shows that in the case of parallel propagation the dispersion relation for ES waves is not affected by the inhomogeneity. 
Another limit to be considered is the case of waves propagating along the direction of inhomogeneity $\left(k_{\|}=0, \sin \psi=0\right)$. In this case, Eq. (40) is reduced to

$$
\begin{gathered}
-i \frac{\omega}{k} \sum_{\alpha} \frac{X_{\alpha}}{n_{\alpha}} \sum_{n=-\infty}^{+\infty} \int d^{3} p p_{\perp} \frac{1}{\gamma_{\alpha} D_{\alpha_{n}}} \frac{n}{b_{\alpha}} \frac{\partial f_{\alpha}^{\prime}}{\partial p_{\perp}} \frac{n J_{n}^{2}}{b_{\alpha}} \\
+1+\frac{\omega^{2}}{k} \sum_{\alpha} \frac{X_{\alpha}}{n_{\alpha}} m_{\alpha} \sum_{n=-\infty}^{+\infty} \int d^{3} p \frac{1}{D_{\alpha_{n}}} \frac{n J_{n}^{2}}{b_{\alpha}} \frac{\partial f_{\alpha}}{\partial p_{\perp}}=0 .
\end{gathered}
$$

It is seen that the effect of inhomogeneity comes entirely from the term with the derivative of the dielectric tensor, introduced in Eq. (37).

Another interesting limit is that of waves propagating perpendicularly both to the magnetic field and to the inhomogeneity $\left(k_{\|}=0, \sin \psi=1\right)$.

$$
\begin{aligned}
& 1+\frac{\omega^{2}}{k} \sum_{\alpha} \frac{X_{\alpha}}{n_{\alpha}} m_{\alpha} \sum_{n=-\infty}^{+\infty} \int d^{3} p \frac{1}{D_{\alpha_{n}}} \frac{n J_{n}^{2}}{b_{\alpha}} \frac{\partial f_{\alpha}}{\partial p_{\perp}} \\
&+ \frac{\omega^{2}}{k} \sum_{\alpha} \frac{X_{\alpha}}{n_{\alpha}} \frac{1}{\Omega_{\alpha}} \sum_{n=-\infty}^{+\infty} \int d^{3} p \frac{1}{D_{\alpha_{n}}} J_{n}^{2} f_{\alpha}^{\prime} \\
&+\frac{\omega^{2}}{k} \sum_{\alpha} \frac{X_{\alpha}}{n_{\alpha}} \frac{1}{\Omega_{\alpha}} \sum_{n=-\infty}^{+\infty} \int d^{3} p p_{\perp} \frac{1}{D_{\alpha_{n}}} \frac{n J_{n}^{2}}{b_{\alpha}} \frac{n}{b_{\alpha}} \frac{\partial f_{\alpha}^{\prime}}{\partial p_{\perp}}=0 .
\end{aligned}
$$

In this case, there are inhomogeneity effects on the dielectric constant. It is seen that, if the medium is homogeneous, this case of propagation perpendicular to the inhomogeneity is the same as the case of propagation parallel to the inhomogeneity, as it should be.

\section{Summary and conclusions}

We have presented a detailed derivation of the effective dielectric tensor for electromagnetic waves propagating in arbitrary direction in a weakly inhomogeneous plasma. Some of the details of the derivation have already appeared in the literature, but were repeated here in order to clearly define the notation, and because they are important in order to show details of the derivation of the particular limiting case for electrostatic fluctuations. The calculations have shown once again that the effective dielectric tensor satisfies Onsager symmetry, and have also corroborated earlier results of the literature which indicated that the symmetry is lost in the case of a limiting form of the tensor derived assuming the absence of magnetic fluctuations. We have therefore argued using basic features of electromagnetic theory that the limiting electrostatic form obtained for the effective dielectric tensor is a spurious result which does not satisfy the whole set of Maxwell equations and does not constitute the correct form of description of dielectric properties for the case of electrostatic fluctuations, which require a dielectric constant. We have then provided a general expression for the dielectric constant which incorporate inhomogeneity effects, derived from the effective dielectric tensor and therefore satisfying energy conservation requirements.

\section{Acknowledgments}

The present research was supported by Brazilian agencies CNPq and FAPERGS. L.F.Z. acknowledges useful discussions with M. Bornatici, and thanks the hospitality of Instituto di Fisica A. Volta, Universitá di Pavia, Italy, during part of the North Hemisphere Winter of 2003.

\section{A Some details on the transition be- tween two expressions for the com- ponents of the dielectric tensor}

In the term with the $\delta_{j y}$ in Eqs. (21) and (30), we have

$$
\left(1-\frac{k_{\|} p_{\|}}{m_{\alpha} \gamma_{\alpha} \omega}\right)-\frac{k_{\perp} p_{\perp}}{m_{\alpha} \gamma_{\alpha} \omega} \frac{n}{b_{\alpha}}
$$

Using the definition of $b_{\alpha}$, this is equal to

$$
\left(1-\frac{k_{\|} p_{\|}}{m_{\alpha} \gamma_{\alpha} \omega}\right)-\frac{n \Omega_{\alpha}}{\gamma_{\alpha} \omega}=\frac{D_{n \alpha}}{\omega} .
$$

When this result is introduced either in Eq. (21) or in Eq. (30), the denominator is cancelled, and the following property can be used to add the Bessel functions which remain.

$$
\sum_{n=-\infty}^{+\infty} \pi_{i}^{*} \pi_{z}=\left(\frac{p_{\|}}{p_{\perp}}\right)^{2} \delta_{i z} .
$$

Moreover, in Eqs. (20) and (21), and also in (30), we have terms with a $\delta_{j z}$ containing the following,

$$
\frac{k_{\perp}}{m_{\alpha} \gamma_{\alpha} \omega} \frac{n}{b_{\alpha}} \mathcal{L}(g)+\frac{\partial g}{\partial p_{\|}},
$$


where $g$ can be $f_{\alpha}$ or $f_{\alpha}^{\prime}$. Using the definition of $b_{\alpha}$, this can be written as

$$
\frac{n \Omega_{\alpha}}{\gamma_{\alpha} \omega} \frac{1}{p_{\perp}} \mathcal{L}(g)+\frac{\partial g}{\partial p_{\|}},
$$

and therefore

$$
\begin{gathered}
\left(1-\frac{k_{\|} p_{\|}}{m_{\alpha} \gamma_{\alpha} \omega}\right) \frac{1}{p_{\perp}} \mathcal{L}(g)+\frac{\partial g}{\partial p_{\|}}-\frac{D_{n \alpha}}{\omega} \frac{1}{p_{\perp}} \mathcal{L}(g), \\
=\frac{p_{\|}}{p_{\perp}} \varphi_{0}(g)-\frac{D_{n \alpha}}{\omega} \frac{1}{p_{\perp}} \mathcal{L}(g),
\end{gathered}
$$

When this result is introduced either in Eq. (20), (21), or in Eq. (30), the term with the $\varphi_{0}$ operator is incorporated into the terms with $\left(1-\delta_{j z}\right)$, and in the other term the denominator is cancelled, and Eq. (43) can be used to add the Bessel functions which remain, along with the following property.

$$
\sum_{n=-\infty}^{+\infty} \Phi_{i}^{*} \pi_{z}=\frac{1}{2} \frac{p_{\|}}{p_{\perp}} \delta_{i y},
$$

The outcome is, in either case, Eq. (25), (26) or Eq. (31).

\section{B The derivation of the effective di- electric constant}

Using Eq. (38) with the components of the effective dielectric tensor as given by Eq. (30), we use the following results,

$$
\begin{gathered}
\sum_{i j} k_{i} \delta_{i j} k_{j}=\sum_{i} k_{i} k_{i}=k^{2}, \\
\sum_{i j} k_{i} \delta_{i z} \delta_{j z} k_{j}=k_{z} k_{z}=k_{\|}^{2}, \\
\sum_{i j} k_{i}\left(\delta_{i y} \delta_{j z}+\delta_{j y} \delta_{i z}\right) k_{j}=2 k_{y} k_{z} \\
=2 k_{\|} k_{\perp} \sin \psi,
\end{gathered}
$$

and obtain after some algebraic manipulations,

$$
\begin{gathered}
\varepsilon_{l}=1+\frac{\omega}{k^{2}} \sum_{\alpha} \frac{X_{\alpha}}{n_{\alpha}} \sum_{n=-\infty}^{+\infty} \int d^{3} p p_{\perp} \frac{1}{\gamma_{\alpha} D_{\alpha_{n}}} \\
\times\left\{\left(\frac{\partial f_{\alpha}}{\partial p_{\perp}}-\frac{k_{\|}}{m_{\alpha} \gamma_{\alpha} \omega} \mathcal{L}\left(f_{\alpha}\right)\right) \sum_{i j} k_{i} \pi_{i}^{*} \pi_{j} k_{j}\right. \\
\left.+\frac{1}{p_{\|}} \mathcal{L}\left(f_{\alpha}\right) \sum_{i} k_{i} \pi_{i}^{*} \pi_{z} k_{\|}\left[-1+\frac{k_{\|} p_{\|}}{m_{\alpha} \gamma_{\alpha} \omega}+\frac{k_{\perp} p_{\perp}}{m_{\alpha} \gamma_{\alpha} \omega} \frac{n}{b_{\alpha}}\right]\right\} \\
+\frac{\omega}{k^{2}} \sum_{\alpha} \frac{X_{\alpha}}{n_{\alpha}} \frac{1}{m_{\alpha} \Omega_{\alpha}} \sum_{n=-\infty}^{+\infty} \int d^{3} p p_{\perp}^{2} \frac{1}{\gamma_{\alpha} D_{\alpha_{n}}} \\
\times\left\{1-\frac{k_{\|} p_{\|}}{m_{\alpha} \gamma_{\alpha} \omega}-\frac{k_{\perp} p_{\perp}}{m_{\alpha} \gamma_{\alpha} \omega} \frac{n}{b_{\alpha}}\right] \frac{1}{p_{\|}} k_{\perp} \sin \psi f_{\alpha}^{\prime} \sum_{i} k_{i} \pi_{i}^{*} \pi_{z} \\
+\frac{k_{\perp} \sin \psi}{m_{\alpha} \gamma_{\alpha} \omega} f_{\alpha}^{\prime} \sum_{i j} k_{i} \pi_{i}^{*} \pi_{j} k_{j} \\
+\left(\frac{\partial f_{\alpha}^{\prime}}{\partial p_{\perp}}-\frac{k_{\|}}{m_{\alpha} \gamma_{\alpha} \omega} \mathcal{L}\left(f_{\alpha}^{\prime}\right)\right) \sum_{i j} k_{i}\left(\Phi_{i}^{*} \pi_{j}\right)^{H} k_{j} \\
\left.+\frac{1}{p_{\|}} \mathcal{L}\left(f_{\alpha}^{\prime}\right) \sum_{i} k_{i}\left(\Phi_{i}^{*} \pi_{z}\right)^{H} k_{\|}\left[-1+\frac{k_{\|} p_{\|}}{m_{\alpha} \gamma_{\alpha} \omega}+\frac{k_{\perp} p_{\perp}}{m_{\alpha} \gamma_{\alpha} \omega} \frac{n}{b_{\alpha}}\right]\right\} .
\end{gathered}
$$

Using the definition of the $\pi_{i}$, we obtain

$$
\begin{gathered}
\sum_{i} k_{i} \pi_{i}=J_{n}\left(\frac{n}{b_{\alpha}} k_{\perp}+k_{\|} \frac{p_{\|}}{p_{\perp}}\right) \\
\sum_{i j} k_{i} \pi_{i}^{*} \pi_{j} k_{j}=J_{n}^{2}\left(\frac{n}{b_{\alpha}} k_{\perp}+k_{\|} \frac{p_{\|}}{p_{\perp}}\right)^{2}
\end{gathered}
$$


We also need the following quantity,

$$
\sum_{i j} k_{i}\left(\Phi_{i}^{*} \pi_{j}\right)^{H} k_{j}=\frac{1}{2}\left[\left(\mathbf{k} \cdot \vec{\Phi}^{*}\right)(\mathbf{k} \cdot \vec{\pi})+\text { c.c. }\right] .
$$

Since the quantity $k_{i} \pi_{i}$ is real,

$$
\frac{1}{2}\left[\left(\mathbf{k} \cdot \vec{\Phi}^{*}\right)(\mathbf{k} \cdot \vec{\pi})+\text { c.c. }\right]=(\mathbf{k} \cdot \vec{\pi})(\mathbf{k} \cdot \operatorname{Re} \vec{\Phi})
$$

Using the definition of the $\Phi_{i}$,

$$
\begin{gathered}
\sum_{i} k_{i} \operatorname{Re} \Phi_{i}=k_{\perp}\left[\frac{n^{2} J_{n}}{b_{\alpha}^{2}}-\frac{J_{n}}{2}-\frac{J_{n}^{\prime}}{b_{\alpha}}\right] \sin (2 \psi) \cos \psi \\
+k_{\perp}\left\{\frac{J_{n}}{2}-\left[\frac{n^{2} J_{n}}{b_{\alpha}^{2}}-\frac{J_{n}}{2}-\frac{J_{n}^{\prime}}{b_{\alpha}}\right] \cos (2 \psi)\right\} \sin \psi \\
+k_{\|} \frac{p_{\|}}{p_{\perp}} \frac{n}{b_{\alpha}} J_{n} \sin \psi \\
=\operatorname{Re} \Phi_{z}^{*}\left(k_{\perp} \frac{p_{\perp}}{p_{\|}} \frac{n}{b_{\alpha}}+k_{\|}\right)-k_{\perp} \frac{J_{n}^{\prime}}{b_{\alpha}} \sin \psi
\end{gathered}
$$

Therefore,

$$
\begin{gathered}
\sum_{i j} k_{i}\left(\Phi_{i}^{*} \pi_{j}\right)^{H} k_{j}=\left(\sum_{i} k_{i} \pi_{i}\right)\left(\sum_{i} k_{i} \operatorname{Re} \Phi_{i}\right) \\
=\left(k_{\perp} \frac{p_{\perp}}{p_{\|}} \frac{n}{b_{\alpha}}+k_{\|}\right) \pi_{z} \sin \psi \\
\times\left[\frac{n}{b_{\alpha}} \pi_{z}\left(k_{\perp} \frac{p_{\perp}}{p_{\|}} \frac{n}{b_{\alpha}}+k_{\|}\right)-k_{\perp} \frac{J_{n}^{\prime}}{b_{\alpha}}\right] .
\end{gathered}
$$

Using Eqs. (50) and (51) in Eq. (48),

$$
\begin{aligned}
\varepsilon_{l}=1+ & \frac{\omega}{k^{2}} \sum_{\alpha} \frac{X_{\alpha}}{n_{\alpha}} \sum_{n=-\infty}^{+\infty} \int d^{3} p p_{\perp} \frac{1}{\gamma_{\alpha} D_{\alpha_{n}}} J_{n}^{2}\left(\frac{n}{b_{\alpha}} k_{\perp}+k_{\|} \frac{p_{\|}}{p_{\perp}}\right) \\
& \times\left\{\left(\frac{\partial f_{\alpha}}{\partial p_{\perp}}-\frac{k_{\|}}{m_{\alpha} \gamma_{\alpha} \omega} \mathcal{L}\left(f_{\alpha}\right)\right)\left(\frac{n}{b_{\alpha}} k_{\perp}+k_{\|} \frac{p_{\|}}{p_{\perp}}\right)\right. \\
& \left.+\mathcal{L}\left(f_{\alpha}\right) \frac{k_{\|}}{m_{\alpha} \gamma_{\alpha} \omega}\left[-\frac{m_{\alpha} \gamma_{\alpha} \omega}{p_{\perp}}+k_{\|} \frac{p_{\|}}{p_{\perp}}+k_{\perp} \frac{n}{b_{\alpha}}\right]\right\} \\
+ & \frac{\omega}{k^{2}} \sum_{\alpha} \frac{X_{\alpha}}{n_{\alpha}} \frac{k_{\perp}}{m_{\alpha} \Omega_{\alpha}} \sum_{n=-\infty}^{+\infty} \int d^{3} p p_{\perp}^{2} \frac{1}{\gamma_{\alpha} D_{\alpha_{n}}} J_{n} \\
& \times\left\{\left[\frac{m_{\alpha} \gamma_{\alpha} \omega}{p_{\perp}}-k_{\|} \frac{p_{\|}}{p_{\perp}}-k_{\perp} \frac{n}{b_{\alpha}}\right] \frac{1}{m_{\alpha} \gamma_{\alpha} \omega} f_{\alpha}^{\prime} J_{n}\right. \\
& \left.+\frac{p_{\|}}{m_{\alpha} \gamma_{\alpha} \omega} f_{\alpha}^{\prime} \frac{k_{\perp}}{k_{\perp}} J_{n}\left(\frac{n}{b_{\alpha}} k_{\perp}+k_{\|} \frac{p_{\|}}{p_{\perp}}\right)\right\} \\
& +\frac{\omega}{k^{2}} \sum_{\alpha} \frac{X_{\alpha}}{n_{\alpha}} \frac{\sin \psi}{m_{\alpha} \Omega_{\alpha}} \sum_{n=-\infty}^{+\infty} \int d^{3} p p_{\perp}^{2} \frac{1}{\gamma_{\alpha} D_{\alpha_{n}}} \\
& \left\{\left(\frac{\partial f_{\alpha}^{\prime}}{\partial p_{\perp}}-\frac{k_{\|}}{m_{\alpha} \gamma_{\alpha} \omega} \mathcal{L}\left(f_{\alpha}^{\prime}\right)\right)\left(k_{\perp} \frac{n}{b_{\alpha}}+k_{\|} \frac{p_{\|}}{p_{\perp}}\right)\right.
\end{aligned}
$$




$$
\begin{aligned}
& \times {\left[\frac{n}{b_{\alpha}} J_{n}^{2}\left(k_{\perp} \frac{n}{b_{\alpha}}+k_{\|} \frac{p_{\|}}{p_{\perp}}\right)-k_{\perp} \frac{J_{n} J_{n}^{\prime}}{b_{\alpha}}\right] } \\
&+\mathcal{L}\left(f_{\alpha}^{\prime}\right) \frac{k_{\|} \sin \psi}{m_{\alpha} \gamma_{\alpha} \omega}\left[\frac{n}{b_{\alpha}} J_{n}^{2}\left(k_{\perp} \frac{n}{b_{\alpha}}+k_{\|} \frac{p_{\|}}{p_{\perp}}\right)-k_{\perp} \frac{J_{n} J_{n}^{\prime}}{b_{\alpha}}\right] \\
&\left.\times\left[-\frac{m_{\alpha} \gamma_{\alpha} \omega}{p_{\perp}}+k_{\|} \frac{p_{\|}}{p_{\perp}}+k_{\perp} \frac{n}{b_{\alpha}}\right]\right\} .
\end{aligned}
$$

After some simple algebraic manipulation, we obtain the following,

$$
\begin{aligned}
\varepsilon_{l} & =1+\frac{\omega}{k^{2}} \sum_{\alpha} \frac{X_{\alpha}}{n_{\alpha}} \sum_{n=-\infty}^{+\infty} \int d^{3} p p_{\perp} \frac{1}{\gamma_{\alpha} D_{\alpha_{n}}} J_{n}^{2} \\
\times & \left(\frac{n}{b_{\alpha}} k_{\perp}+k_{\|} \frac{p_{\|}}{p_{\perp}}\right)\left[k_{\perp} \frac{n}{b_{\alpha}} \frac{\partial f_{\alpha}}{\partial p_{\perp}}+k_{\|} \frac{\partial f_{\alpha}}{\partial p_{\|}}\right] \\
+ & \frac{\omega}{k^{2}} \sum_{\alpha} \frac{X_{\alpha}}{n_{\alpha}} \frac{k_{\perp}}{m_{\alpha} \Omega_{\alpha}} \sum_{n=-\infty}^{+\infty} \int d^{3} p p_{\perp} \frac{1}{\gamma_{\alpha} D_{\alpha_{n}}} \\
+ & \left.\frac{\omega}{k^{2}} \sum_{\alpha} \frac{X_{\alpha}}{n_{\alpha}} \frac{\sin \psi}{m_{\alpha} \Omega_{\alpha}} \sum_{n=-\infty}^{+\infty} \int \frac{n}{b_{\alpha}} k_{\perp}+k_{\|} \frac{p_{\|}}{p_{\perp}}\right) f_{\alpha}^{\prime} p p_{\perp}^{2} \frac{1}{\gamma_{\alpha} D_{\alpha_{n}}} \\
& \times\left[\frac{n J_{n}^{2}}{b_{\alpha}}\left(k_{\perp} \frac{n}{b_{\alpha}}+k_{\|} \frac{p_{\|}}{p_{\perp}}\right)-k_{\perp} \frac{J_{n} J_{n}^{\prime}}{b_{\alpha}}\right] \\
\times & {\left[k_{\perp} \frac{n}{b_{\alpha}} \frac{\partial f_{\alpha}^{\prime}}{\partial p_{\perp}}+k_{\|} \frac{\partial f_{\alpha}^{\prime}}{\partial p_{\|}}\right] . }
\end{aligned}
$$

It is important to notice that none of the components $k_{i}$ labeled as coming from the magnetic fluctuations have survived up to this point.

We now write

$$
\begin{gathered}
\left(\frac{n}{b_{\alpha}} k_{\perp}+k_{\|} \frac{p_{\|}}{p_{\perp}}\right) f_{\alpha}^{\prime}=\frac{m_{\alpha} \gamma_{\alpha}}{p_{\perp}}\left(\frac{n \Omega_{\alpha}}{\gamma_{\alpha}}+\frac{k_{\|} p_{\|}}{m_{\alpha} \gamma_{\alpha}}\right) \\
=\frac{m_{\alpha} \gamma_{\alpha}}{p_{\perp}}\left(\omega-D_{\alpha_{n}}\right) .
\end{gathered}
$$

Inserting this expression into Eq. (52), and using $\sum_{n} n J_{n}^{2}=0, \sum_{n} J_{n}^{2}=1$ and $\sum_{n} n^{2} J_{n}^{2}(x)=x^{2} / 2$, and also taking into account that

we obtain Eq. (39).

$$
\int d^{3} p \partial f_{\alpha} / \partial p_{\|}=0, \quad \int d^{3} p p_{\perp} \frac{\partial f_{\alpha}^{\prime}}{\partial p_{\perp}}=-2 \int d^{3} p f_{\alpha}^{\prime},
$$

\section{References}

[1] A. B. Mikhailovskii, in Oscillations of an inhomogeneous plasma, Vol. 3 of Reviews of Plasma Physics (Consultants Bureau, New York, 1967), pp. 159-227, M. A. Leontovich, editor.

[2] A. B. Mikhailovskii, in Instabilities in inhomogeneous plasma, Vol. 1: Basic Plasma Physics I of Handbook of Plasma Physics (North Holland, Amsterdam, 1983), Chap. 3.4, pp. 587-610, M. N. Rosenbluth and R. Z. Sagdeev, general editors; A. A. Galeev and R. N. Sudan, editors.

[3] A. B. Mikhailovskii and O. G. Onishchenko, J. Plasma Phys. 37, 15 (1987).
[4] H. P. Freund, D. Dillenburg, and C. S. Wu, J. Plasma Phys. 27, 69 (1982).

[5] R. A. Caldela Filho, R. S. Schneider, and L. F. Ziebell, J. Plasma Phys. 42, 165 (1989).

[6] V. S. Beskin, A. V. Gurevich, and Y. I. Istomin, Sov. Phys. JETP 65, 715 (1987).

[7] M. Bornatici and Y. A. Kravtsov, Plasma Phys. Contr. Fusion 42, 255 (2000).

[8] R. Gaelzer, R. S. Schneider, and L. F. Ziebell, Phys. Rev. E 51, 2407 (1995).

[9] R. Gaelzer, R. S. Schneider, and L. F. Ziebell, Phys. Rev. E 55, 5859 (1997). 
[10] R. Gaelzer, L. F. Ziebell, and O. J. G. Silveira, Phys. Plasmas 6, 4533 (1999)

[11] O. J. G. Silveira, L. F. Ziebell, R. Gaelzer, and P. H. Yoon, Phys. Rev. E 65, 036407, 11p. (2002).

[12] C. N. Lashmore-Davies and R. O. Dendy, Phys. Fluids B 4, 493 (1992)

[13] D. C. McDonald, R. A. Cairns, and C. N. Lashmore-Davies, Phys. Plasmas 1, 842 (1994).
[14] C. N. Lashmore-Davies and R. O. Dendy, Phys. Fluids B 1, 1565 (1989).

[15] C. N. Lashmore-Davies and R. O. Dendy, Phys. Rev. Lett. 62, 1982 (1989).

[16] R. A. Cairns et al., Phys. Fluids B 3, 2953 (1991).

[17] M. Nambu, Phys. Plasmas 3, 4325 (1996).

[18] T. H. Stix, The theory of plasma waves (AIP, New York, 1992). 